\title{
Influencia de la altitud sobre la estructura, composición florística y carbono de bosques del Chocó
}

\author{
Jhon Jerley Torres-Torres ${ }^{1}(\mathbb{D})$, Víctor Eleazar Mena-Mosquera ${ }^{(D)}$ \& Melissa Niyet Rueda-Sánchez ${ }^{2}$ (D)
}

1. Universidad Tecnológica del Chocó “Diego Luis Córdoba”, Facultad de Ingeniería, Grupo de Investigación Ciencia Animal y Recursos Agroforestales, Contratista Vicerrectoría de Investigaciones, Quibdó, Colombia, A. A. 292 B/ Nicolás Medrano, Quibdó, Colombia; i-jhon.torres@utch.edu.co (Autor para correspondencia)

2. Universidad Tecnológica del Chocó "Diego Luis Córdoba", Facultad de Ingeniería, Grupo de Investigación Agroforestería del Trópico Húmedo Chocoano, Quibdó, Colombia, victor.mena@utch.edu.co, memovie@gmail.com, menyrusa_1720@hotmail.com

Recibido 21-IX-2021 • Corregido 16-XI-2021・ Aceptado 01-XII-2021

DOI: https://doi.org/10.22458/urj.v14i1.3746

\begin{abstract}
Influence of altitude on the structure, floristic composition and carbon of Choco forests". Introduction: Despite the wide biodiversity found in the Chocó, little has been documented on the influence of altitude on floristic composition, structure, diversity, biomass and tree carbon. Objective: To evaluate the influence of altitude on the floristic composition, structure and carbon stored in forests of the Quibdo (QDO)Carmen de Atrato (CA) altitudinal gradient in the biogeographic Choco. Methods: Eighteen plots $(20 \times 20 \mathrm{~m})$ were established in a gradient of 100-1700m altitude between QDO and CA, Choco. The floristic composition was analyzed using Ward's method and a simple linear regression was performed to observe the relationship between altitude and structural and biomass variables. Diversity was estimated using Shannon's and Simpson's indices. Biomass was calculated using an allometric equation developed for this forest type. Carbon was estimated with a carbon fraction of 0,5 . Results: A total of 773 individuals were found, comprising 90 species and 33 botanical families. The sites at lower altitudes presented greater richness. The families with the highest number of tree species were: Fabaceae, Malvaceae, Lauraceae and Clusiaceae. Ward's analysis allowed separating the sites into two statistically different groups $(p<0,05)$, the first of lower altitude dominated by the species Iryanthera tricornis Ducke, Brosimum utile (Kunth) Pittier. and Pithecellobium sp. and the second of higher altitude dominated by Aniba puchury-minor (Mart.) Mez. At the density level, few differences were observed between sites, while the basal area decreased with increasing altitude. For the variables species richness, basal area, diversity and biomass, negative relationships and correlations were obtained with altitude, while for density this was positive. On average, carbon stored in aboveground biomass was $68.28 \mathrm{Mg} \mathrm{h}$ 1 for the QDO- CA altitudinal gradient (100-1700masl), which decreases as altitude increases and differs statistically among the sites sampled $(p<0,05)$. Conclusion: Altitude exerts a strong negative influence on the floristic composition (species richness and floristic turnover), structure (basal area and Importance Value Index), biomass and carbon of the ecosystems studied.
\end{abstract}

Keywords: Tree biomass, natural forests, Biogeographic Choco, Climate change, Floristic diversity, Floristic diversity.
RESUMEN. Introducción: A pesar de la amplia biodiversidad que se presenta en el Chocó, es poco lo que se ha documentado sobre la influencia de la altitud en la composición florística, estructura, diversidad, biomasa y carbono arbóreo. Objetivo: Evaluar la influencia de la altitud sobre la composición florística, estructura y carbono almacenado en bosques del gradiente altitudinal Quibdó (QDO)-Carmen de Atrato (CA) en el Chocó biogeográfico. Métodos: Establecimos 18 parcelas $(20 \times 20 \mathrm{~m})$ distribuidas en un gradiente de 100-1700m de altitud comprendida entre QDO y CA, Chocó. La composición florística fue analizada mediante el método de Ward y se realizó una regresión lineal simple para observar la relación entre la altitud y las variables estructurales y de biomasa. La diversidad fue estimada a través de los índices de Shannon y Simpson. Se calculó la biomasa empleando una ecuación alométrica desarrollada para este tipo de bosque. El carbono fue estimado con una fracción de carbono de 0,5. Resultados: Encontramos un total de 773 individuos, comprendido en 90 especies y 33 familias botánicas. Los sitios de menor altitud presentaron mayor riqueza. Las familias con mayor número de especies arbóreas fueron: Fabaceae, Malvaceae, Lauraceae y Clusiaceae. El análisis de Ward permitió separar los sitios en dos grupos diferentes estadísticamente $(p<0,05)$, el primero de menor altitud dominado por las especies Iryanthera tricornis Ducke, Brosimum utile (Kunth) Pittier. y Pithecellobium sp. y el segundo de mayor altitud dominado por Aniba puchuryminor (Mart.) Mez. A nivel de densidad se observaron pocas diferencias entre los sitios, mientras que el área basal se reduce conforme aumenta la altitud. Para las variables riqueza de especie, área basal, diversidad y biomasa se obtuvieron relaciones y correlaciones negativas con la altitud, mientras que para la densidad esta fue positiva. En promedio se obtuvo un carbono almacenado en la biomasa aérea de $68,28 \mathrm{Mg} \mathrm{h}^{-1}$ para el gradiente altitudinal QDO- CA (100-1700msnm), el cual disminuye conforme aumenta la altitud y difiere estadísticamente entre los sitios muestreados $(p<0,05)$. Conclusión: La altitud ejerce una fuerte influencia negativa en la composición florística (riqueza de especie y recambio florístico), la estructura (área basal e Índice de Valor de Importancia) biomasa y carbono de los ecosistemas estudiados.

Palabras clave: Biomasa arbórea, bosques naturales, Chocó Biogeográfico, cambio climático, diversidad florística. 
Los bosques tropicales son considerados los ecosistemas terrestres más importantes del planeta, debido a su extensión geográfica, complejidad ecológica, alta biodiversidad y tasas de endemismo (Myers et al., 2000, Pan et al., 2013). Así mismo, debido a la alta tasa de captura y almacenamiento de carbono atmosférico, ejercen un rol fundamental en el balance del carbono global y en la mitigación del calentamiento global (Pan et al., 2013; Intergovernmental Panel on Climate Change [IPCC], 2014). Bajo este contexto, Pan et al. (2013) en sus análisis reportaron que estos bosques naturales presentan la mayor biomasa arbórea (aérea + subterránea) con un promedio de $327,8 \mathrm{Mg} \mathrm{h}^{-1}$ (Pan et al. 2013). Igualmente, algunas investigaciones desarrolladas en las últimas décadas han indicado que los bosques húmedos y lluviosos tropicales de baja altitud, pueden presentar una biomasa aérea arbórea de entre 96,3 y $541,6 \mathrm{Mg} \mathrm{h}^{-1}$, la cual suele relacionarse con la composición del ecosistema y factores ambientales como la altitud (Chave et al., 2008; TorresTorres et al., 2017).

La profunda variación en la composición, estructura y la biomasa aérea registrada en bosques tropicales se debe en gran medida al hecho de que esta es dinámica y puede verse influenciada significativamente por fenómenos climáticos (precipitación, temperatura y humedad), biológicos (composición de especies, tipo de especie, estructura, grupo ecológico) y ambientales (altitud, edad del sustrato, fertilidad del suelo, topografía); además, integra procesos como el establecimiento de plantas, crecimiento, mortalidad, sucesión y disturbios (Asner et al., 2009; Poorter et al., 2016). Asner et al. (2009) observaron que la composición de especies dominantes y la biomasa aérea se encuentran determinadas por la precipitación, altitud y edad del sustrato en bosques Iluviosos tropicales en Hawaii (Asner et al., 2009). Mientras que, Oberleitner et al. (2021) reportaron que la biomasa aérea y estructura florística se encuentran relacionadas con la edad del sustrato, la cobertura boscosa y la riqueza de especies en bosques de Costa Rica. Los señalamientos anteriores sugieren una significativa importancia de los factores ambientales (altitud, clima), ecológicos (diversidad) y biológicos (composición y estructura) sobre la acumulación de biomasa aérea y carbono en bosques tropicales.

En un análisis a escala de bosques Neotrópicales, Poorter et al. (2015) indicaron que la composición florística, estructura y biomasa aérea de los árboles está fuertemente explicada por la altitud, el diámetro promedio de los árboles y la diversidad de especies; mientras que, la fertilidad del suelo presentó una débil relación con la biomasa. Por tal razón, y basados en los resultados de Asner et al. (2009) y Oberleitner et al. (2021) se presume que la altitud presenta una fuerte correlación con la composición, estructura, diversidad y contenido de carbono de los bosques tropicales con altos niveles de pluviosidad. A pesar de estos resultados, en el Chocó Biogeográfico aún se ignora la influencia que puede tener la altitud sobre la composición florística, estructura, biomasa y carbono de estos ecosistemas (Álvarez-Dávila et al., 2016); información que podría ser un aporte para explicar el patrón de distribución de la flora departamental y nacional. Además, esta información resultaría ser fundamental para la planificación del manejo de estos ecosistemas estratégicos (Mena-Mosquera et al., 2020).

El Chocó biogeográfico, a parte de su alta biodiversidad y endemismo (Myers et al., 2000), tienen una importante función en la mitigación del cambio climático global, pues cuentan con un área boscosa de $\approx 7,8$ millones de hectáreas en Colombia, de las cuales el $80 \%$ son bosques naturales (primarios y secundarios). Sin embargo, en estos ecosistemas las comunidades rurales realizan constantemente actividades antrópicas como tala de árboles y minería a cielo abierto de oro y platino, con lo cual, se destruyen y degradan entre cerca de 360 hectáreas de bosque cada año (Instituto de Investigaciones Ambientales del Pacífico [IIAP], 2015), lo que reduce considerablemente la biomasa aérea de estos sitios, y su capacidad de contribuir con la mitigación 
del cambio climático global, por lo que se hace necesario el emprendimiento de actividades que promueva la conservación y/o aprovechamiento sostenible de estos bosques.

Por consiguiente, se efectuó la presente investigación, para la que se plantearon los siguientes interrogantes: ¿Cuál es la influencia de la altitud en la composición y estructura florística del gradiente altitudinal Quibdó (QDO) - Carmen de Atrato (CA)?, ¿Cuál es la relación existente entre la biomasa arbórea y la altitud en el gradiente QDO-CA?, ¿Qué cantidad de carbono se encuentra almacenado en la biomasa aérea de los bosques del gradiente QDO-CA?

\section{MATERIALES Y MÉTODOS}

Área de estudio. El estudio se realizó entre febrero y marzo del 2020, en el gradiente altitudinal formado entre los municipios de Quibdó y Carmen de Atrato en el Chocó Biogeográfico. Esta área corresponde a las zonas de vida Bosque Pluvial Tropical y Bosque Húmedo Tropical, localizada entre los $5^{\circ} 43^{\prime} 22,11^{\prime \prime}$ de latitud Norte y los $76^{\circ} 19^{\prime} 38,60^{\prime \prime}$ de longitud Oeste, entre los 100 y $1700 \mathrm{msnm}$, con diferencia altitudinales entre sitio de $200 \mathrm{~m}$. La temperatura de los sitios varía entre los 12 y $28^{\circ} \mathrm{C}$ a lo largo del gradiente. La humedad relativa es superior al $70 \%$ y su precipitación media anual se encuentra comprendida entre 2000 y $10000 \mathrm{~mm}$ (Fig. 1).

Los muestreos de vegetación se realizaron en nueve sitios con diferencias altitudinales de $200 \mathrm{~m}$ (Fig. 1), los cuales se seleccionaron teniendo en cuenta su distribución homogénea, es decir, buscando que la diferencia entre sitio fuera igual.

Los ecosistemas seleccionados corresponden a bosques secundarios con uso anterior de vegetación primaria natural, donde predominan especies arbóreas de las familias Fabaceae y Malvaceae.

Toma de datos. En los puntos seleccionados (Fig. 1), se establecieron 18 Parcelas Temporales de Muestreo (PTM) de $20 \times 20 \mathrm{~m}\left(400 \mathrm{~m}^{2}\right)$. A cada individuo por especie se le midió el Diámetro a la Altura del Pecho (DAP a $1,30 \mathrm{~m}$ ) en centímetros $(\mathrm{cm})$ con una cinta diamétrica y la altura en metros $(\mathrm{m})$ con una vara de madera graduada de $15 \mathrm{~m}$ de longitud (Torres-Torres et al., 2016). Para la identificación de los individuos se realizaron colecta de material vegetal, el cual fue comparado con muestras botánicas del Herbario Chocó de la Universidad Tecnológica del Chocó, donde además se depositaron los ejemplares con estructura reproductiva en buen estado. Posterior a esto, se verificaron la vigencia del nombre científico y la familia en Colombia (http://www.biovirtual.unal.edu.co/ICN/), Missouri Botanical Garden (http://www.tropicos.org) New York Botanical Garden (http://www.nybg.org/), Trópicos (http://www.tropicos.org) y The International Plant Names Index (http://www.ipni.org).

Análisis de la composición y estructura de los ecosistemas muestreados. La composición florística en los ecosistemas muestreados fue separada a través de un análisis de agrupamiento realizado a partir de los datos de abundancia y dominancia relativa, utilizando el método de distancias de Ward.

Se realizó una regresión lineal simple para observar la relación entre la altitud, la riqueza de especie, área basal y biomasa. Se calculó el coeficiente de correlación cuadrado $\left(r^{2}\right)$ de Pearson y el valor empírico $p$-valor para el ajuste lineal. Todas estas estimaciones se realizaron en el programa PAST versión 3.1 (Hammer et al., 2001).

La diversidad fue estimada a través de los índices de Shannon y Simpson. La estructura fue analizada por el área basal $\left(\mathrm{m}^{2} \mathrm{ha}^{-1}\right)$, densidad (número de individuos por hectárea) e Índice de Valor de Importancia - IVI (Figueredo et al., 2017). 


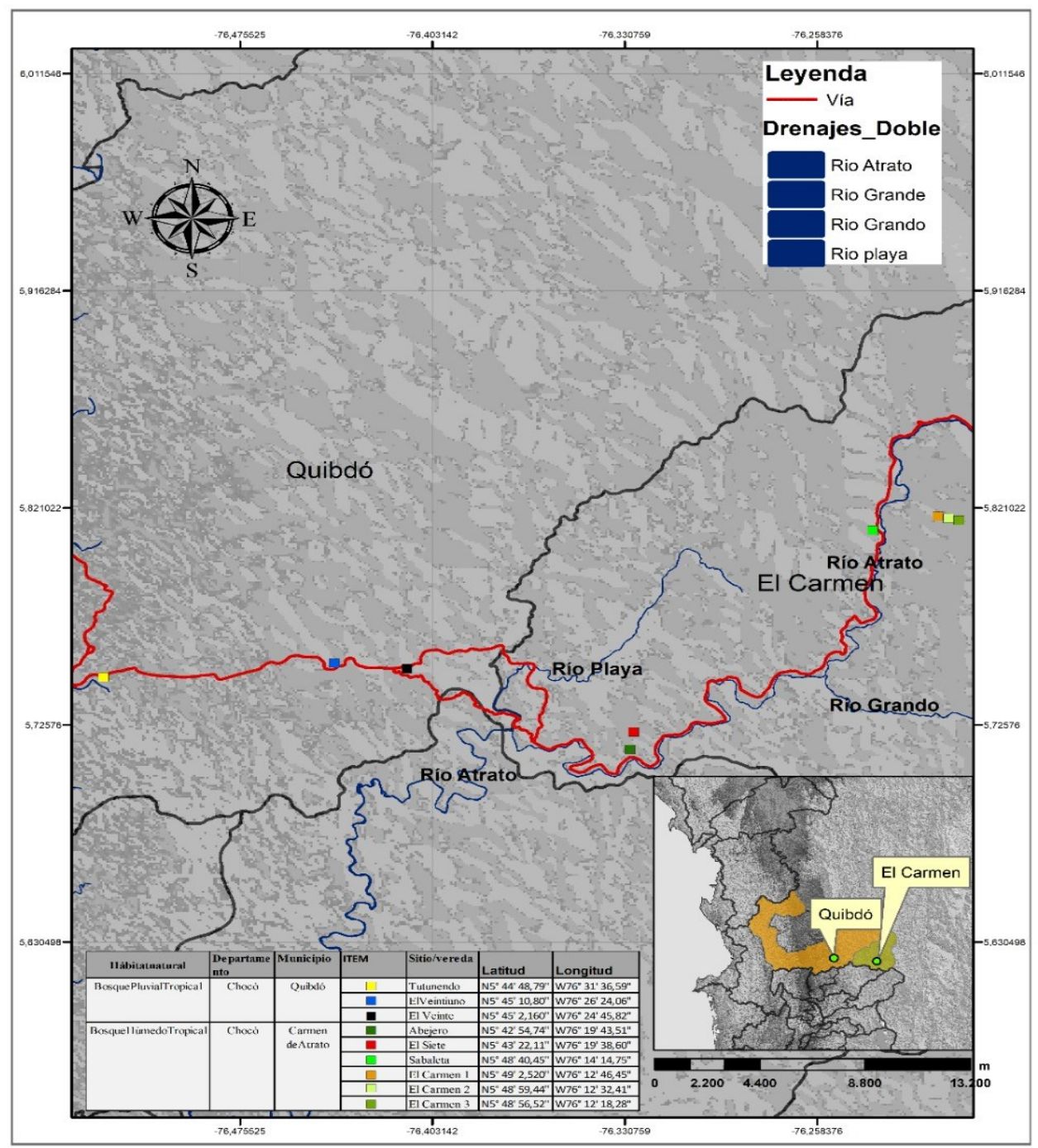

Fig. 1. Localización del área de estudio

Estimación de biomasa y carbono. Para el cálculo de la biomasa aérea se asignaron valores de densidad de la madera a las especies registradas en el censo. Este valor fue extraído de la Base Global de Datos de Densidad de la Madera (BGDM) (Zanne et al., 2009). Finalmente, se estimó la biomasa utilizando la ecuación de Álvarez et al. (2012) desarrollada para este tipo de ecosistemas (Ecuación 1).

$$
\text { Biomasa }=\exp (-2,857+2,081 * \operatorname{In} D+0,587 * \operatorname{In} A l+0,453 * \operatorname{In} D m
$$

Donde:

$D=$ Diámetro.

$A l=$ Altura total .

Dm = Densidad de la madera

El carbono almacenado en la biomasa aérea fue estimado al multiplicar esta última por una fracción de 0,5 (Brown \& Lugo, 1982). Las variables analizadas fueron diversidad, biomasa aérea y carbono. Para determinar la diferencia de en estas variables en los sitios muestreados se realizó un análisis de varianza ANOVA seguido de la prueba de Tukey al 5\% de significancia. 


\section{RESULTADOS}

Composición florística. Se registraron 33 familias, 90 especies y 773 individuos en las 18 parcelas establecidas (Tabla 1). Los sitios de muestreo con mayor cercanía a la cabecera municipal de Quibdó (Tutunendo, El Veintiuno, El Veinte, Abejero y Sabaleta) presentaron un mayor número de especies y mayor riqueza que los sitios más cercanos al Carmen de Atrato.

El Veintiuno fue el sitio con mayor abundancia específica (promedio $=53,5$ individuos por parcela), seguido de Tutunendo (49,5 individuos) y el Veinte $(46,5$ individuos) correspondiendo a las localidades más cercanas a la capital del departamento del Chocó.

Las familias con mayor número de especies fueron: Fabaceae, Malvaceae, Lauraceae y Clusiaceae (Tabla 1). Las especies más abundantes fueron Aiouea dubia Mez con 55 individuos, Brosimum utile (Kunth) Pittier (34), Licania durifolia Cuatrec. (33), Cespedesia spathulata (Ruiz \& Pav.) Planch. (31) y Pithecellobium sp. (30).

En la tabla se relacionan las familias y especies más abundantes del gradiente altitudinal * DM: Densidad de la Madera proveniente de Base Global de Datos de Densidad de la Madera (Zanne et al., 2009)

El análisis de agrupamiento basado en la similitud de la composición florística por medio del valor de cobertura de las especies separó los nueve sitios de muestreo en dos grupos: en el primero las localidades del Veintiuno y Veinte son similares y ligeramente diferentes a Tutunendo y Abejero. Opuestamente con mayor dispersión se ubicaron los sitios de muestreo Carmen 1, Sabaleta, el Siete, Carmen 2 y Carmen 3. Por tanto, difieren a grandes rasgos de los cuatro sitios con menor altitud (los del primer grupo, Fig. 2).

Estructura. Para los sitios con altitud mayor o igual a 1100msnm (Sabaleta, Carmen 1, Carmen 2 y Carmen 2) se destaca la especie Aniba puchury-minor (Mart.) Mez (Laurel), como la de mayor importancia ecológica (IVI entre 14 y 25,30); mientras que para los ecosistemas por debajo de $1100 \mathrm{~m}$ se registraron las especies Pouteria amygdalicarpa, Iryanthera tricornis Ducke, Brosimum utile (Kunth) Pittier. y Pithecellobium sp., como las de mayor IVI (Entre 13,02 y 20,36) (Tabla 2).

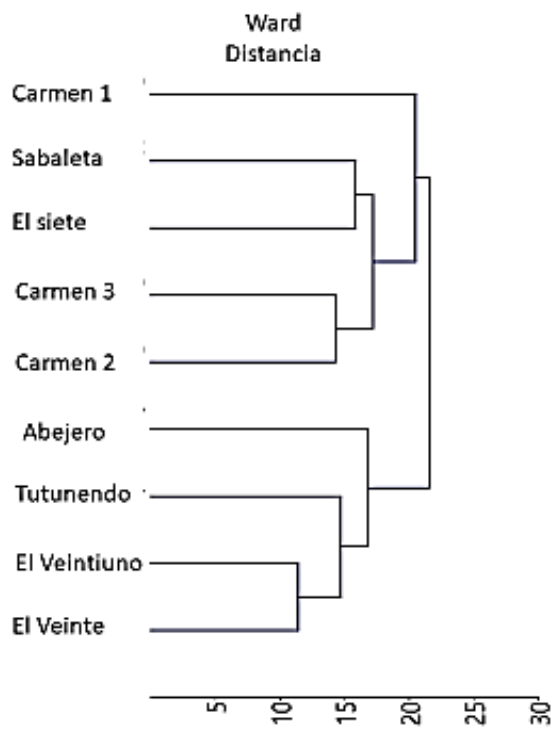

Fig. 2. Dendrograma de agrupamiento de los sitios de muestreo según el valor de cobertura de las especies y la altitud de las zonas 
TABLA 1

Composición florística de nueve sitios de muestreo del gradiente altitudinal Quibdó- Carmen de Atrato

\begin{tabular}{|c|c|c|c|c|}
\hline Familia & Especie & Nombre común & $\begin{array}{l}\text { № de } \\
\text { individuos }\end{array}$ & DM* \\
\hline \multirow{13}{*}{ Fabaceae } & Pithecellobium sp. & Guamo macho & 30 & 0,50 \\
\hline & Dialium guianense (Aubl.) Sandwith & Hueso & 26 & 0,87 \\
\hline & Hymenaea oblongifolia Huber. & Algarrobo & 26 & 0,74 \\
\hline & Pentaclethra macroloba (Willd.) Kuntze & Aserrín & 20 & 0,67 \\
\hline & Hymenolobium sp. & Tostao & 16 & 0,67 \\
\hline & Inga nobilis Willd. & Guamo Rosario & 15 & 0,56 \\
\hline & Orphanodendron bernalii Barneby \& Grimes & Tiratete & 9 & 0,68 \\
\hline & Dipteryx oleifera Benth & Choibá & 6 & 0,91 \\
\hline & Clathrotropis brachypetala (Tul.) Kleinhoonte & Sapan & 5 & 0,81 \\
\hline & Inga acrocephala Steud. & Guamillo & 5 & 0,51 \\
\hline & Machaerium seemannii Benth. & Bálsamo & 3 & 0,59 \\
\hline & Copaifera canime Harms & Canime & 2 & 0,61 \\
\hline & Andira inermis (Wright) DC. & Aji amargo & 1 & 0,66 \\
\hline \multirow{8}{*}{ Malvaceae } & Ochroma pyramidale (Cav.) Urb. & Balso & 19 & 0,14 \\
\hline & Apeiba glabra Aubl. & Peinemono & 14 & 0,32 \\
\hline & Huberodendron patinoi Cuatrec. & Carrá & 11 & 0,50 \\
\hline & Phragmotheca hydra sp. & Aguacatón & 5 & 0,44 \\
\hline & Quararibea sp. & Ahuyamo & 5 & 0,50 \\
\hline & Hampea punctulata Cuatrec. & Algodoncillo & 1 & 0,44 \\
\hline & Trichilia quadrijuga Kunth & Cedro macho & 22 & 0,55 \\
\hline & Trichilia sp. & Chagará & 5 & 0,64 \\
\hline \multirow{8}{*}{ Lauraceae } & Aiouea dubia Mez & Laurel & 55 & 0,37 \\
\hline & Aniba perutilis Hemsl. & Chachajo & 8 & 0,50 \\
\hline & Nectandra sp. & Caidita & 5 & 0,54 \\
\hline & Ocotea calophylla Mez & Laurel blanco & 4 & 0,50 \\
\hline & Ocotea cernua (Nees) Mez & Jigua baba & 3 & 0,32 \\
\hline & Ocotea sp. & Laurel mono & 3 & 0,50 \\
\hline & $\begin{array}{l}\text { Caryodaphnopsis inaequalis (A.C.Sm.) van der } \\
\text { Werff \& Richt. }\end{array}$ & Incibe & 2 & 0,60 \\
\hline & Nectandra aff. Longifolia & Tuave canelo & 2 & 0,54 \\
\hline \multirow{5}{*}{ Clusiaceae } & Calophyllum longifolium Willd. & Aceite & 10 & 0,58 \\
\hline & Clusia grandiflora Splitg. & Madroño & 8 & 0,60 \\
\hline & Symphonia globulifera L.fil. & Tometo & 4 & 0,62 \\
\hline & Tovomita sp. & Chagualo & 4 & 0,68 \\
\hline & Chrysochlamys clusiifolia Maguire & Manteco & 1 & 0,43 \\
\hline $\begin{array}{l}\text { Otras } \\
\text { familias } \\
\text { (29) }\end{array}$ & ( & 355 & & \\
\hline Total & 90 & 773 & & \\
\hline
\end{tabular}

El ecosistema localizado a 1100msnm (Carmen 1), resultó ser el de mayor densidad, mientras que se observa que los ecosistemas de menor altitud contienen mayor área basal (Fig. 4A y 4B). Al comparar la riqueza de especie se obtuvo que las localidades Tutunendo, Sabaleta y Carmen 2 son diferentes estadísticamente con respecto a los otros sitios muestreados; mientras que para la diversidad no se observaron diferencias estadísticas (Shannon $-p=0,63$; Simpson $-p=0,46$, Tabla 3 ). 
TABLA 2

Especies con mayor IVI en función a los sitios muestreados

\begin{tabular}{|c|c|c|c|c|c|}
\hline Sitio (m.s.n.m.) & Nombre científico & $\operatorname{Ar}(\%)$ & $\operatorname{Fr}(\%)$ & $\mathrm{Da}(\%)$ & IVI \\
\hline & Pentaclethra macroloba (Willd.) Kuntze & 10,10 & 4,76 & 0,50 & 15,36 \\
\hline \multirow[t]{3}{*}{ Tutunendo (100) } & Ochroma pyramidale (Cav.) Urb. & 2,02 & 2,38 & 12,28 & 16,68 \\
\hline & Pouteria amygdalicarpa & 11,11 & 4,76 & 4,49 & 20,36 \\
\hline & Hymenaea oblongifolia Huber. & 4,72 & 3,51 & 3,49 & 11,71 \\
\hline \multirow[t]{3}{*}{ El Veintiuno (300) } & Iryanthera tricornis Ducke & 7,55 & 3,51 & 1,96 & 13,02 \\
\hline & Brosimum utile (Kunth) Pittier & 5,66 & 1,75 & 4,70 & 12,12 \\
\hline & Pouteria amygdalicarpa & 5,43 & 3,85 & 3,95 & 13,23 \\
\hline \multirow[t]{3}{*}{ El Veinte (500) } & Trichilia quadrijuga Kunth & 4,35 & 3,85 & 4,32 & 12,51 \\
\hline & Apeiba glabra Aubl. & 4,35 & 3,85 & 4,28 & 12,47 \\
\hline & Couma macrocarpa Barb.Rodr. & 3,23 & 4,00 & 5,79 & 13,02 \\
\hline \multirow[t]{3}{*}{ Abejero (700) } & Cespedesia spathulata (Ruiz \& Pav.) Planch. & 6,45 & 4,00 & 3,33 & 13,78 \\
\hline & Brosimum utile (Kunth) Pittier & 9,68 & 4,00 & 4,55 & 18,23 \\
\hline & Protium heptaphyllum (Aubl.) Marchand & 4,40 & 3,77 & 5,04 & 13,21 \\
\hline \multirow[t]{3}{*}{ El siete (900) } & Coumasp. & 6,59 & 3,77 & 2,50 & 12,87 \\
\hline & Pithecellobium sp. & 6,59 & 3,77 & 4,34 & 14,71 \\
\hline & Aniba puchury-minor (Mart.) Mez & 7,41 & 4,35 & 2,25 & 14,00 \\
\hline \multirow[t]{3}{*}{ Sabaleta (1100) } & Cespedesia spathulata (Ruiz \& Pav.) Planch. & 4,94 & 4,35 & 4,66 & 13,95 \\
\hline & Prumnopitys montana Humb. \& Bonpl. ex Willd. & 6,17 & 4,35 & 3,16 & 13,68 \\
\hline & Inga nobilis Willd. & 11,39 & 8,00 & 4,70 & 24,09 \\
\hline \multirow[t]{3}{*}{ Carmen 1 (1300) } & Dialium guianense (Aubl.) Sandwith & 10,13 & 8,00 & 5,92 & 24,05 \\
\hline & Aniba puchury-minor (Mart.) Mez & 13,92 & 8,00 & 3,37 & 25,30 \\
\hline & Pithecellobium $s p$. & 13,43 & 5,56 & 1,72 & 20,70 \\
\hline \multirow[t]{3}{*}{ Carmen 2 (1500) } & Pourouma cecropiifolia Mart. & 5,97 & 5,56 & 2,87 & 14,40 \\
\hline & Aniba puchury-minor (Mart.) Mez & 20,90 & 5,56 & 6,77 & 33,22 \\
\hline & Aniba puchury-minor (Mart.) Mez & 9,84 & 6,06 & 2,63 & 18,53 \\
\hline \multirow[t]{2}{*}{ Carmen 3 (1700) } & Socratea exorrhiza (Mart.) H.Wendl. & 4,92 & 6,06 & 6,76 & 17,74 \\
\hline & Hymenolobium sp. & 9,84 & 6,06 & 3,55 & 19,45 \\
\hline Total & & 100 & 100 & 100 & 300 \\
\hline
\end{tabular}

Ar: Abundancia relativa; Fr: Frecuencia relativa; Dr: Dominancia relativa; IVI: Índice de Valor de Importancia

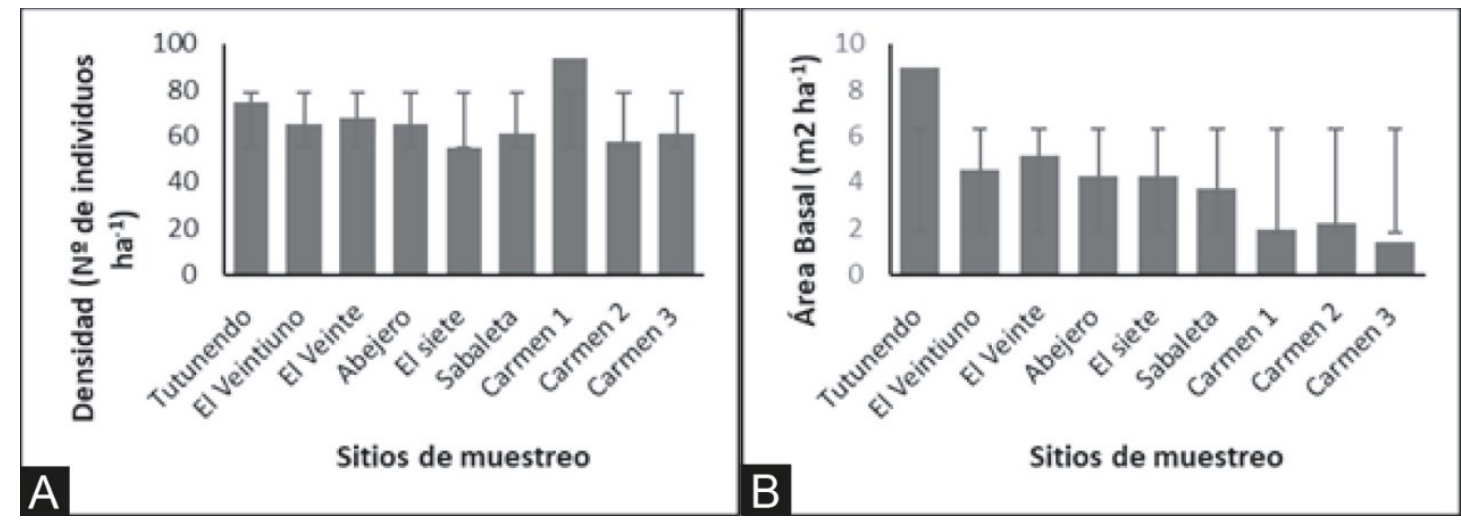

Fig. 4. Promedio de la densidad (A) y área basal (B) registrados en las unidades de muestreo del gradiente altitudinal Quibdó - Carmen de Atrato 


\section{TABLA 3}

Riqueza e índices de diversidad de Shannon y Simpson para los sitios muestreados en el gradiente altitudinal Quibdó Carmen de Atrato (promedio \pm desviación estándar)

\begin{tabular}{lccccccccc}
\hline & \multicolumn{7}{c}{ Sitio de muestreo } \\
\cline { 2 - 9 } Índice & Tutunendo & $\begin{array}{c}\text { El } \\
\text { Veintiuno }\end{array}$ & El Veinte & Abejero & El siete & Sabaleta & Carmen 1 & Carmen 2 & Carmen 3 \\
\hline Riqueza & $33,0 \pm 0,2 \mathrm{~b}^{*}$ & $40,0 \pm 0,1 \mathrm{a}$ & $37,0 \pm 0,2 \mathrm{ab}$ & $36,0 \pm 0,2 \mathrm{ab}$ & $41,0 \pm 0,1 \mathrm{a}$ & $33,0 \pm 0,2 \mathrm{~b}$ & $21,0 \pm 0,2 \mathrm{c}$ & $29,0 \pm 0,2 \mathrm{~b}$ & $25,0 \pm 0,2 \mathrm{c}$ \\
Shannon & $3,2 \pm 0,1 \mathrm{a}$ & $3,5 \pm 0,0 \mathrm{a}$ & $3,4 \pm 0,0 \mathrm{a}$ & $3,3 \mathrm{a}$ & $3,5 \pm 0,0 \mathrm{a}$ & $3,3 \pm 0,0 \mathrm{a}$ & $2,9 \pm 0,1 \mathrm{a}$ & $3,0 \pm 0,1 \mathrm{a}$ & $3,1 \pm 0,1 \mathrm{a}$ \\
Simpson & $0,9 \pm 0,1 \mathrm{a}$ & $0,9 \pm 0,1 \mathrm{a}$ & $0,9 \pm 0,1 \mathrm{a}$ & $0,9 \pm 0,1 \mathrm{a}$ & $0,9 \pm 0,1 \mathrm{a}$ & $0,9 \pm 0,1 \mathrm{a}$ & $0,9 \pm 0,1 \mathrm{a}$ & $0,9 \pm 0,1 \mathrm{a}$ & $0,9 \pm 0,1 \mathrm{a}$ \\
\hline
\end{tabular}

Los valores corresponden al promedio \pm desviación estándar.

*Letras diferentes indican diferencias estadísticas entre los ecosistemas muestreados

Se encontraron correlaciones inversas y significativas entre la altitud y dos de las variables estructurales (riqueza de especie y área basal) (Tabla 4), lo que indica que conforme aumenta la altitud el valor de estas disminuye (Fig. 5). Del mismo modo, se halló una correlación inversa no significativa entre la altitud y la diversidad de los ecosistemas (Tabla 4).

De otro lado, la correlación entre la altitud y la densidad fue directa y sin diferencias significativas ( $p=0,564$; Tabla 4$)$.

Biomasa y carbono aéreo. Se obtuvo una relación negativa entre la biomasa y la altitud ( $r=$ $-0,89$; Fig. $5 C)$, la cual es significativamente diferente ( $p=0,007$; Tabla 4).

En promedio se obtuvo un carbono almacenado en la biomasa aérea de $68,28 \mathrm{Mg} \mathrm{h}^{-1}$ para el gradiente altitudinal Quibdó - Carmen de Atrato, el cual tiende a ser menor conforme aumenta la altitud. El contenido de carbono de los sitios ubicados por debajo de los $1100 \mathrm{msnm}$ es estadísticamente diferente al obtenido a los sitios de mayor altitud ( $p<0,05$, Tabla 5 ).

TABLA 4

Correlación entre la altitud y variables estructurales, diversidad y Biomasa de los ecosistemas muestreado

\begin{tabular}{lcc}
\multicolumn{1}{c}{ Variables } & r-Pearson* & $p$ \\
\hline Riqueza & $-0,68$ & 0,003 \\
Densidad (No de individuos ha-1 $)$ & 0,06 & 0,564 \\
Área basal $\left(\mathrm{m}^{2} \mathrm{ha}^{-1}\right)$ & $-0,89$ & 0,002 \\
Shannon & $-0,62$ & 0,632 \\
Simpson & $-0,57$ & 0,457 \\
Biomasa (Mg ha-1) & $-0,89$ & 0,007 \\
\hline
\end{tabular}

* r-Pearson: correlación de Pearson; $p<0,05$ indica que hay diferencia estadísticamente significativa entre los sitios muestreados con respecto a las variables 

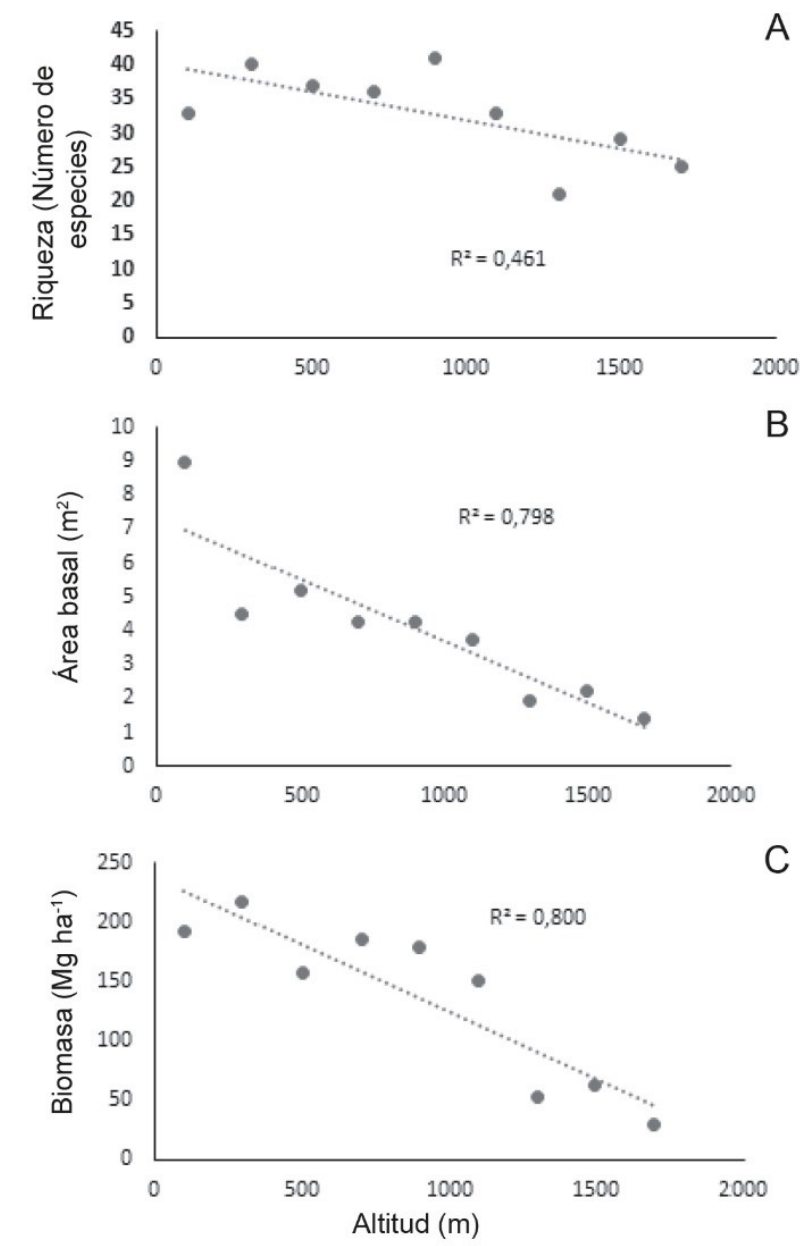

Fig. 5. Relación entre la altitud con la riqueza (A), Área basal (B) y Biomasa (C) en los sitios de muestreo del gradiente altitudinal Quibdó - Carmen de Atrato

TABLA 5

Carbono almacenado en la biomasa aérea estimado por sitio de muestreo en el gradiente altitudinal Quibdó - Carmen de Atrato

\begin{tabular}{lcc}
\hline $\begin{array}{c}\text { Sitio de muestreo } \\
\text { (m.s.n.m.) }\end{array}$ & Biomasa (Mg h-1) & Carbono (Mg h ${ }^{-1}$ ) \\
\hline Tutunendo (100) & $192 \pm 21,2 \mathrm{a}^{*}$ & $96 \pm 21,2 \mathrm{a}$ \\
El Veintiuno (300) & $217,34 \pm 26,5 \mathrm{a}$ & $108,67 \pm 26,5 \mathrm{a}$ \\
El Veinte (500) & $157,33 \pm 15,1 \mathrm{ab}$ & $78,67 \pm 15,1 \mathrm{ab}$ \\
Abejero (700) & $185,91 \pm 19,2 \mathrm{a}$ & $92,96 \pm 19,2 \mathrm{a}$ \\
El siete (900) & $179,44 \pm 13,1 \mathrm{a}$ & $89,72 \pm 13,1 \mathrm{a}$ \\
Sabaleta (1100) & $150,57 \pm 9,1 \mathrm{ab}$ & $75,29 \pm 9,1 \mathrm{ab}$ \\
Carmen 1(1300) & $53,26 \pm 6,6 \mathrm{c}$ & $26,63 \pm 6,6 \mathrm{c}$ \\
Carmen 2 (1500) & $62,95 \pm 7,4 \mathrm{c}$ & $31,48 \pm 7,4 \mathrm{c}$ \\
Carmen 3 (1700) & $30,18 \pm 10,3 \mathrm{c}$ & $15,09 \pm 10,3 \mathrm{c}$ \\
Promedio & & 68,21 \\
\hline
\end{tabular}

$1 \mathrm{Mg}=10^{6} \mathrm{~g}$. Los valores corresponden al promedio \pm desviación estándar. Letras diferentes indican diferencias estadísticas entre los ecosistemas muestreados 


\section{DISCUSIÓN}

Composición florística. Los resultados de esta investigación (90 especies en 0,7 ) corroboran que el Chocó es una de las regiones más ricas en especies vegetales (Galeano, 2001; Asprilla et al., 2003; García et al., 2004), siendo estos resultados superiores a los reportados en estudios realizados para esta región (Quinto-Mosquera \& Moreno, 2014; Torres-Torres et al., 2016; Mena-Mosquera et al., 2020). Torres-Torres et al. (2016) registraron 83 especies vegetales en 0,9ha; Casas et al. (2018) 59 especies en 0,5ha y Mena-Mosquera et al. (2020) 71 especies en $2 \mathrm{ha}$. Sin embargo, los resultados obtenidos se encuentran por debajo del rango sugerido por Galeano (2001) y Rangel (2004) para ecosistemas del Chocó Biogeográfico (150 -250). Estas diferencias pueden estar relacionadas con la disminución de individuos que se experimenta a medida que aumenta la altitud (Alvear et al., 2010). El número de familias, especies e individuos registrados en este estudio es mayor al encontrado en el páramo de Anaime, Tolima (Colombia) por Segura et al. (2019), quienes encontraron 22 familias y 27 especies, sin desconocer que estos autores muestrearon áreas por encima de los $2000 \mathrm{msnm}$, lo que podría apoyar la teoría que indica que, a mayor elevación, menor riqueza específica.

Los resultados de abundancia, frecuencia y dominancia de los sitios muestreados sugieren menores valores en los sitios de mayor altitud, lo cual es similar a los hallazgos de Macía \& Fuertes (2008), quienes registraron 196 especies en un gradiente comprendido entre 1340-1385 en Bolivia, donde el número de especies disminuyó conforme se asciende en altitud. De otro lado, estos resultados difieren de los obtenidos por Cueva et al. (2019), quienes hallaron mayores especies, familias e individuos en zonas por encima de los 2160msnm. Estas diferencias pueden ser explicadas por la intensa dinámica de la vegetación provocada por los deslizamientos de laderas por causas naturales, lo que hace que los individuos de mayor porte se disminuyan en las partes más elevadas (Oberleitner et al., 2021; Giacomotti et al., 2021).

Se encontraron un promedio de 35 especies por sitio de muestreo y con una disminución significativa conforme aumenta la altitud. Esta baja puede estar asociada a factores como la topografía, la altitud o el suelo. Pudiendo ser principalmente la altitud un factor limitante en la disminución del número de especies arbóreas para estos bosques. A una determinada altitud por existir condiciones climáticas más adversas, pocas especies arbóreas podrían estar presentes (Giacomotti et al., 2021).

De las 90 especies forestales distribuidas en las 18 parcelas se destacan A. dubia, B. utile, L. durifolia, C. spathulata y Pithecellobium sp., las cuales son características de bosques naturales húmedos tropicales del Chocó Biogeográfico (Quinto-Mosquera \& Moreno, 2014; Torres-Torres et al., 2016; Palacios-Tello et al., 2017; Mena-Mosquera et al., 2020).

Mediante la separación de los nueve sitios de muestreo con base en la similitud de la composición florística a lo largo del gradiente QDO - CA se obtuvieron dos grupos que difieren estadísticamente conforme aumenta la altitud. Este resultado corrobora el planteamiento de Cabrera et al. (2019), quienes sugieren que la altitud es el primer factor jerárquico, responsable de la división de grupos estructurales y florísticos.

Los resultados de esta investigación sugieren una correlación negativa entre la altitud y la riqueza florística. Este hallazgo difiere de lo indicado por Linares (2008) y Loza et al. (2010) en bosques de niebla localizados por encima de los $2000 \mathrm{~m}$ de altitud en Venezuela y Bolivia, en los que encontraron relaciones positivas entre la altitud y la diversidad.

Estructura. El análisis estructural de los ecosistemas muestreados señala similitudes en la densidad de ocho de los sitios, sin embargo, al aumentar la altitud se encontró menor área basal. Este resultado difiere de los obtenidos por Cueva et al. (2019) y Segura et al. (2019), quienes reportaron mayor área basal en sitios con mayor altitud. Estas diferencias pueden hallar explicación 
en factores ambientales como la temperatura, humedad, precipitación y fertilidad del suelo (Asner et al., 2009), por lo que se debe explorar esta posibilidad mediante otras investigaciones. También, este contraste en la composición y estructura florística de estos ecosistemas se deben a gradientes ecológicos (altitud) y geomorfológicos (litología y paisaje) (Cabrera et al., 2019). Sin embargo, se podrían presentar cambios locales de precipitación, humedad, y velocidad del viento que también pueden explicar la variación de diversidad y composición con un cambio de altitud cada $200 \mathrm{~m}$ (Siles et al., 2017).

Los resultados estructurales ratifican y explican parte de la hipótesis planteada en esta investigación, comprobándose cambios ligeros en la vegetación conforme se asciende a nivel altitudinal, sin embargo, no se evidenciaron diferencias entre la altitud y la diversidad. Estos hallazgos sugieren que los bosques húmedos y pluviales tropicales ubicados en el gradiente altitudinal QDO - CA la riqueza de especie disminuye conforme aumenta la altitud, lo cual es similar a lo documentado por Girardin et al. (2014).

Biomasa y Carbono aéreo. Se encontró un carbono almacenado en la biomasa aérea promedio de $68,21 \mathrm{Mg} \mathrm{h}^{-1}(613,85 \mathrm{Mg}$ en $0,72 \mathrm{ha})$. Este resultado difiere del obtenido en bosques húmedos y pluviales tropicales del Chocó biogeográfico (Torres-Torres et al., 2017; QuintoMosquera \& Moreno, 2017; Mena-Mosquera, 2020); por lo que se crea la necesidad de avanzar en estudios que permitan comprender la dinámica de estos ecosistemas.

Se logra observar que la altitud ejerce una influencia negativa en el proceso de captura y almacenamiento de carbono en la biomasa de la vegetación. Este resultado contrasta con el obtenido en investigaciones realizadas en los andes (Lozano et al., 2010; Cuevas et al., 2019; Segura et al., 2019), en las que se ha indicado que los valores de biomasa aumentan conforme se asciende en altitud. Estas diferencias podrían explicarse por la tesis de Wadsworth (2000), quien expresa que la riqueza y el contenido de biomasa registrado en altas elevaciones podría deberse a la influencia de zonas de transición (Ecotono).

Estadísticamente los bosques de menor altitud almacenaron mayor carbono en la biomasa aérea de la vegetación arbórea en comparación con los de mayor altitud, encontrándose valores en el rango sugerido por Phillips et al. (2011), para bosques por encima de los $800 \mathrm{msnm}$ en Colombia (48,1 a 129,4Mg ha $\left.{ }^{-1}\right)$, sin embargo, se debe analizar la relación entre la diversidad arbórea e los sitios y su Productividad Primaria Neta, con la intención de dilucidar como es la dinámica de estos ecosistemas.

Los bajos valores de carbono almacenado en la biomasa aérea de los sitios con mayor altitud contrastan con lo sugerido por Segura et al. (2019), quienes indican que, a pesar de las bajas temperaturas de estos sitios, la vegetación arbórea retiene gran cantidad de carbono en su biomasa aérea, por su misma adaptación al clima del sitio.

Los bosques más importantes para el almacenamiento de carbono y preservación con miras a mitigación del cambio climático son los de baja altitud, lo cual corrobora lo documentado por Chave et al. (2008), quienes además señalan que el contenido de carbono suele relacionarse con la composición florística del sitio y factores ambientales como la altitud.

En conclusión, se evidencia que la altitud ejerce una fuerte influencia negativa en la composición florística (riqueza de especie y recambio florístico), la estructura (área basal e IVI) biomasa y carbono de los ecosistemas estudiados. De otro lado, se debe considerar que bajo las condiciones de estudio la densidad no es fuertemente influenciada por la altitud, siendo esto explicado por la poca diferencia en la cantidad de individuos de los sitios muestreados. De igual forma, no se evidenciaron diferencias a nivel de diversidad alfa. 
La diversidad florística de los ecosistemas muestreados se correlaciona de forma negativa no significativa con la altitud; por lo que se infiere que para esta parte del Chocó Biogeográfico la altitud no es un condicionante directo de la diversidad específica arbórea.

El carbono almacenado en la biomasa aérea se correlaciona de forma negativa y significativa con la altitud, lo que indica que el tema altitudinal si afecta la acumulación de biomasa, por lo que se requeriría de nuevas investigaciones en las que se determine como varia la tasa fotosintética en el área muestreada (100-1700msnm).

\section{AGRADECIMIENTOS}

La investigación es producto del proyecto "Diversidad florística en bosques naturales de la gradiente altitudinal Quibdó - Carmen de Atrato, Colombia-Contrato 408", formulado y ejecutado por el Grupo de Investigación Agroforestería del Trópico Húmedo Chocoano (AGROTROPICO) de la Universidad Tecnológica del Chocó (UTCH). Los autores agradecen a las comunidades asentadas en el área de influencia del proyecto por su apoyo en la fase de campo, a la Vicerrectoría de Investigación de la UTCH por la financiación parcial del primer autor; de igual forma, a Haminson Mena-Mena por su apoyo en la estimación de parte de los estadígrafos presentados en el manuscrito y a Fausto Córdoba por la elaboración del mapa del área de estudio.

\section{ÉTICA, CONFLICTO DE INTERESES Y DECLARACIÓN DE FINANCIAMIENTO}

Declaramos haber cumplido con todos los requisitos éticos y legales pertinentes, tanto durante el estudio como en la preparación de este documento; que no hay conflictos de interés de ningún tipo, y que todas las fuentes financieras se detallan plena y claramente en la sección de agradecimientos. Asimismo, estamos de acuerdo con la versión editada final de esta publicación. El respectivo documento legal firmado se encuentra en los archivos de la revista.

La declaración de contribución de cada autor es la siguiente: J. J. T. T. Escribió el primer borrador del manuscrito, realizó el análisis estadístico de la investigación y retroalimentó los borradores del artículo; V. E. M. M. Formuló y Gestionó el proyecto del que se originó el manuscrito, revisó y retroalimentó los informes de investigación y los borradores del artículo; M. N. R. S. Ilevó a cabo la investigación en terreno.

\section{REFERENCIAS}

Álvarez, E., Duque, A., Saldarriaga, J., Cabrera, K., De las Salas, G. Del Valle, I., Lema, A., Moreno, F. Orrego, S., \& Rodríguez, L. (2012). Tree above-ground biomass allometries for carbon stocks estimation in the natural forests of Colombia. Forest Ecology and Management, 267, 297-308. https://doi.org/10.1016/j.foreco.2011.12.013

Álvarez-Dávila, E., Jaramillo, G. C., Cogollo, C. C., Martínez. H., Rojas, E., \& Fernández, F. (2016). Structure and diversity of the three plant associations in the San Juan river delta, Chocó, Colombia. Revista Ávore, 40(5), 833-843. https://doi.org/10.1590/0100-67622016000500007

Alvear, M., Betancur, J., \& Franco-Rosselli, P. (2010). Diversidad florística y estructura de remanentes de bosque andino en la zona de amortiguación del Parque Nacional Natural los Nevados, cordillera central colombiana. Caldasia, 32(1), 39-63.

Asner, G. P., Hughes, R. F., Varga, T. A., Knapp, D. E., \& Kennedy-Bowdoin, T. (2009). Environmental and biotic controls over aboveground biomass throughout a tropical rainforest. Ecosystems, 12(2), 261-278. https://doi.org/10.1007/s10021-008-9221-5 
Asprilla, A., Mosquera, C., Valoyes, H., Cuesta, H., \& García, F. (2003). Composición florística de un bosque pluvial tropical (bp-T) en la parcela permanente de investigación en biodiversidad (PPIB) en Salero, Unión Panamericana, Chocó. En F. García, Y. Ramos, J. Palacios, E. Arroyo, A. Mena \& M. González (Eds.), Salero: Diversidad Biológica de un Bosque Pluvial Tropical (bp-T) (1ra ed., pp. 39-44). Editora Guadalupe Ltda.

Brown, S., \& Lugo, A. E. (1982). The storage and production of organic matter in tropical forests and their role in de global carbon cycle. Biotropica, 14(3), 161-187.

Cabrera, O., Benítez, Á., Cumbicus, N., Naranjo, C., Ramón, P., Tinitana, F., \& Escudero, A. (2019). Geomorphology and Altitude Effects on the Diversity and Structure of the Vanishing Montane Forest of Southern Ecuador. Diversity, 11(3), 2-15. https://doi.org/10.3390/d11030032

Casas, L. F., González, D. M., Segura, B., Mosquera, R. A., \& Álvarez, E. (2018). Diversidad y estructura de bosques contrastantes en la región del Chocó-Darién, Colombia. Revista de Investigación Agraria y Ambiental, 9(2), 1727. https://doi.org/10.22490/21456453.2328

Chave, J., Condit, R., Muller-Landau, H. C., Thomas, S. C., Ashton, P. S., Bunyavejchewin, S., Co, L.L., Dattaraja, H., Davies, S., Esufali, S., Ewango, C.E.N., Feeley, K.J., Foster, R.B., Gunatillete, N., Gunatillete, S., Hall, P., Hart, T.B., Hernandez, C., Hubbell, P.S., Itoh, A., ... Losos, E.C. (2008). Assessing evidence for a pervasive alteration in tropical tree communities. PLoS Biology, 6(3), e45. https://doi.org/10.1371/journal.pbio.0060045

Cuevas, E., Lozano, D., \& Yaguana, C. (2019). Efecto de la gradiente altitudinal sobre la composición florística, estructura y biomasa arbórea del bosque seco andino, Loja, Ecuador. Bosque (Valdivia), 40(3), 365-378. http://dx.doi.org/10.4067/S0717-92002019000300365

Figueredo, L. M., Acosta, F., \& Blanco, J. (2017). Composición florística y estructura horizontal del bosque semideciduo micrófilo de la reserva ecológica Siboney-Juticí, Cuba, Rodriguésia, 68(2), 315-324. http://doi.org/10.1590/21757860201768203

Galeano, G. (2001). Estructura, riqueza y composición de plantas leñosas en el golfo de Tribugá, Chocó-Colombia. Caldasia, 23(1), 213-236.

García, F. J., Moreno, M., Robledo, D., Mosquera, L., \& Palacios, L. (2004). Composición y diversidad florística de los bosques de la cuenca hidrográfica del río Cabí, Quibdó-Chocó. Investigación Biodiversidad y Desarrollo, 20(1), 13-23.

Giacomotti, J., Reynel, C., Fernández-Hilario, R., Revilla, I., Palacios-Ramos, S., Terreros-Camac, S., Daza, A., \& LinaresPalomino, R. (2021). Diversidad y composición florística en un gradiente altitudinal en Chanchamayo, selva central del Perú. Folia Amazónica, 30(1), 1-14. https://doi.org/10.24841/fa.v30i1.533

Girardin, C., Farfan-Rios, W. Garcia, K., Feeley, K. J., Jørgensen, P. M., Murakami, A. A., Pérez, L. C., Seidel, R., Paniagua, N., Claros, A. F., Maldonado, C., Silman, M., Salinas, N., Reynel, C., Neill, D. A., Serrano, M., Caballero, C. J., la Torre Cuadros, M. de los A., Macía, M., Killeen, T. J., Malhi, Y. (2014). Spatial patterns of above-ground structure, biomass and composition in a network of six Andean elevation transects. Plant Ecology \& Diversity, 7(1-2), 161171. https://doi.org/10.1080/17550874.2013.820806

Hammer, Ø., Harper, A. T., \& Ryan, P. D. (2001). PAST: Paleontological Statistics Software Package for Education and Data Analysis. Palaeontologia Electronica, 4(1), 1-9. https://palaeo-electronica.org/2001_1/past/past.pdf

Instituto de Investigaciones Ambientales del Pacífico (IIAP). (2015). Plan Integral de Cambio Climático / Chocó-Colombia. Ministerio de Ambiente y Desarrollo Sostenible. https://bit.ly/3ygeHow

Intergovernmental Panel on Climate Change (IPCC). (2014). Climate Change 2014: Synthesis Report. Contribution of Working Groups I, II and III to the Fifth Assessment Report of the Intergovernmental Panel on Climate Change. Core Writing Team, R.K. Pachauri \& L.A. Meyer (Eds.). https://bit.ly/3oA8dNS 
Linares, A. M. (2008). Análisis florístico y estructural de la vegetación de una selva nublada en un gradiente altitudinal, en la Mucuy, Estado de Mérida [Tesis de doctorado, Universidad de los Andes]. SaberULA Repositorio Institucional de la Universidad de Los Andes. https://bit.ly/3IKw7EH

Loza, I., Moraes, M., \& Jørgensen, P. M. (2010). Variación de la diversidad y composición florística en relación a la elevación en un bosque montano boliviano (PNANMI Madidi). Ecología en Bolivia, 45(2), 87-100.

Lozano, P., Küppers, M., \& Bussmann, R. W. (2010). Diversity of paramo and andean elfin forest in Podocarpus National Park - Loja, Ecuador. Arnaldoa, 17(2), 193-202.

Macía, M., \& Fuertes, J. (2008). Composición florística y estructura de los árboles en un bosque tropical montano de la Cordillera Mosetenes, Bolivia. Revista Boliviana de Ecología y Conservación Ambiental, 23, 1-14.

Mena-Mosquera, V. E. (2020). Valoración del servicio ambiental de almacenamiento y fijación de carbono en bosques y sistemas agroforestales con cacao en territorios de comunidades afrodescendientes del departamento del Chocó. [Tesis de doctorado, Universidad de la Salle]. Repositorio institucional. https://bit.ly/3lybZzb

Mena-Mosquera, V. E., Andrade, H. J., \& Torres-Torres, J. J. (2020). Composición florística, estructura y diversidad del bosque pluvial tropical de la subcuenca del río Munguidó, Quibdó, Chocó, Colombia. Entramado, 16(1), $204-215$ https://doi.org/10.18041/1900-3803/entramado.1.6109

Myers, N., Mittermeier, R. A., Mittermeier, C. G., Da Fonseca, G. A. B., \& Kent, J. (2000). Biodiversity hotspots for conservation priorities. Nature, 403, 853-858. https://doi.org/10.1038/35002501

Oberleitner, F., Egger, C., Oberdorfer, S., Dullinger, S., Wanek, W., \& Hietz, P. (2021). Recovery of aboveground biomass, species richness and composition in tropical secondary forests in SW Costa Rica. Forest Ecology and Management, 479, 118580. https://doi.org/10.1016/j.foreco.2020.118580

Palacios-Tello, L. Y., Perea, K., Bellido, D. M., Caicedo, H. Y., \& Abadia D. (2017). Estructura poblacional de ocho especies maderables amenazadas en el departamento del Chocó-Colombia. Cuadernos de Investigación UNED, 9(1), 107114. https://doi.org/10.22458/urj.v9i1.1685

Pan, Y., Birdsey, R. A., Phillips, O. L., \& Jackson, R. B. (2013). The Structure, Distribution, and Biomass of the World's Forests. Annual Review of Ecology, Evolution, and Systematics, 44(1), 593-622. https://doi.org/10.1146/annurev-ecolsys110512-135914

Phillips, J. F., Duque, A.J., Yepes, A. P., Cabrera, K. R., García, M. C., Navarrete, D. A., Álvarez, E., \& Cárdenas, D. (2011). Estimación de las reservas actuales (2010) de carbono almacenadas en la biomasa aérea en bosques naturales de Colombia. Estratificación, alometría y métodos análiticos. Instituto de Hidrología, Meteorología, y Estudios Ambientales - IDEAM-. https://bit.ly/3EJu6QG

Poorter, L., Bongers, F., Aide, T. M., Zambrano, A.M.A., Balvanera, P., Becknell, J.M., Boukili, V., Brancalion, P. H. S., Broadbent, E. N., Chazdon, R. L., Craven, D., Almeida-Cortez, J. S. de., Cabral, G. A. L., Jong, B. H. J. de., Denslow, J. S., Dent, D. H., DeWalt, S. J., Dupuy, J. M., Durán, S. M., Espírito-Santo, M. M., ... Rozendaal, D. (2016). Biomass resilience of Neotropical secondary forests. Nature, 530(7589), 211-214. https://doi.org/10.1038/nature16512

Poorter, L., van der Sande, M.T., Thompson, J., Arets, E.J., Alarcón, A., Álvarez-Sánchez, J., Ascarrunz, N., Balvanera, P., Barajas-Guzmán, G., Boit, A., Bongers, F., Carvalho, F.A., Casanoves, F., Cornejo-Tenorio, G., Costa, F.R., de Castilho, C.V., Duivenvoorden, J.F., Dutrieux, L.P., Enquist, B.J., Fernández-Méndez, F., ... Peña-Claros, M. (2015). Diversity enhances carbon storage in tropical forests. Global Ecology and Biogeography, 24(11), 1314-1328. https://doi.org/10.1111/geb.12364

Quinto-Mosquera, H., \& Moreno, F. (2014). Diversidad florística arbórea y su relación con el suelo en un bosque Pluvial Tropical del Chocó Biogeográfico. Revista Ávore, 38(6), 1123-1132. https://doi.org/10.1590/S010067622014000600017 
Quinto-Mosquera, H., \& Moreno, F. (2017). Net Primary Productivity and Edaphic Fertility in Two Pluvial Tropical Forests in the Chocó Biogeographical Region of Colombia, PLoS ONE, 12(1), e0168211. https://doi.org/10.1371/journal.pone.0168211

Rangel, J. O. (Ed.). (2004). Colombia Diversidad Biótica IV: El Chocó Biogeográfico/Costa Pacífica. Instituto de Ciencias Naturales, Universidad Nacional de Colombia.

Segura, M. A., Andrade, H. J., \& Mojica, C. A. (2019). Estructura, composición florística y almacenamiento de carbono en bosques nativos del páramo de Anaime, Tolima, Colombia. Ciencia Forestal, 29(1), 157-168. https://doi.org/10.5902/1980509826551

Siles, P., Talavera, P. Andino, F. Alaniz, L., \& Ortiz, W. (2017). Composición florística, estructura y biomasa de los bosques de pino-encino en la reserva Santa Rosa, Tisey, Estelí, Nicaragua. Revista de Biología Tropical, 65(2), 763-775. http://dx.doi.org/10.15517/rbt.v65i2.22928

Torres-Torres, J. J., Mena-Mosquera, V. E., \& Álvarez-Dávila, E. (2016). Composición y diversidad florística de tres bosques húmedos tropicales de edades diferentes, en el Jardín Botánico del Pacífico, municipio de Bahía Solano, Chocó, Colombia. Revista Biodiversidad Neotropical, 6(1), 12-21.

Torres-Torres, J. J., Mena-Mosquera, V. E., \& Álvarez-Dávila, E. (2017). Carbono aéreo almacenado en tres bosques del Jardín Botánico del Pacífico, Chocó, Colombia. Entramado, 13(1), 200-209. https://doi.org/10.18041/entramado.2017v13n1.25110

Wadsworth, F. (2000). Capítulo 3: Los bosques primarios y su productividad. En F. Wadswotrh (Ed.), Producción Forestal para América Tropical (pp. 69-111). Departamento de Agricultura de los Estados Unidos-USDA.

Zanne, A. E., López-González, G., Coomes, D. A., Ilic, J., Jansen, S., Lewis, S. L., Miller, R. B., Swenson, N. G., Wiemann, M. C., \& Chave, J. (2009). Data from: Towards a worldwide wood economics spectrum. Dryad, Dataset. https://doi.org/10.5061/dryad.234 